\title{
Electrospun microfibres with temperature sensitive iridescence from encapsulated cholesteric liquid crystal $\dagger$
}

\author{
Eva Enz $^{a}$ and Jan Lagerwall ${ }^{* a}$
}

\author{
Received Xth $X X X X X X X X X X 20 X X$, Accepted Xth $X X X X X X X X X 20 X X$ \\ First published on the web Xth $X X X X X X X X X X 200 X$ \\ DOI: 10.1039/b000000x
}

We apply coaxial electrospinning to produce core-sheath polymer composite fibres with encapsulated short-pitch cholesteric liquid crystal, giving the fibres iridescent colours due to selective reflection within a narrow band of the visible wavelength spectrum. By modifying the feed rate of the liquid crystal during spinning we can tune the fibre diameter from the sub micron range to about $7 \mu \mathrm{m}$, other ranges being accessible via further modifications of the spinning parameters. We demonstrate that the thinnest fibres display quantised colours, determined primarily by the core diameter, whereas the thicker fibres allow a quasicontinuous change in colour if the cholesteric helix pitch changes. Because of the strong response function of liquid crystals, phases as well as structures changing in response to small changes in the environment, the resulting non-woven fibre mats have potential for smart textiles, in particular in sensing applications.

\section{Introduction}

Electrospinning is a versatile process for producing nano- and microfibres via electrostatic means ${ }^{1-4}$. A polymer solution is pumped through a spinneret (a metallic capillary) kept at high voltage with respect to a grounded collector plate, charging the solution to the extent that electrostatic forces overcome the surface tension. A liquid jet is then ejected which due to electrostatic self-repulsion experiences strong whipping and stretching, reducing its diameter greatly. The solvent evaporates in the process, such that a solid, exceptionally thin fibre is eventually deposited on the collector. Depending on the spinning conditions fibres of different morphology and with varying diameter can be produced. By introducing a second capillary inside the spinneret (Fig. 1), carrying a fluid immiscible with the outer polymer solution, fibres with a well-defined core-shell structure can be produced, a variation referred to as coaxial electrospinning ${ }^{5-9}$.

In this work we use coaxial electrospinning to produce composite microfibres with polymer sheath and a core consisting of a short-pitch cholesteric liquid crystal, giving the fibres entirely new optical properties as well as a responsiveness to external influences. Of equal interest is that the composite fibres provide a versatile way of investigating strong confinement effects on the liquid crystal and its phase transitions, as it is encapsulated in a very narrow cylinder that approaches a 1D

$\dagger$ Electronic Supplementary Information (ESI) available: calculation of $\mathrm{N}^{*}$ pitch and reflection wavelength in fibers. See DOI: 10.1039/b000000x/

a Martin-Luther-University Halle-Wittenberg, Institute of Chemistry Physical Chemistry, von-Danckelmann-Platz 4, 06120 Halle, Germany. Fax: $\quad+49$ (0)345 5527400; Tel: +49 (0)345 5525836; E-mail: jan.lagerwall@lcsoftmatter.com

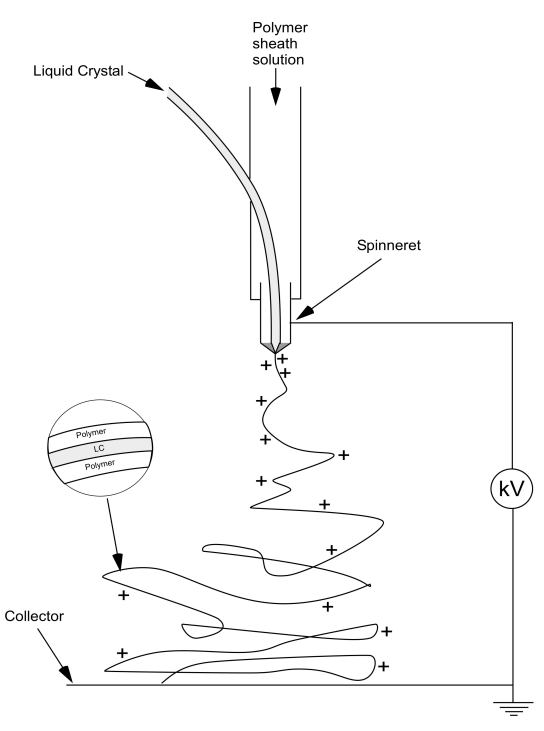

Fig. 1 Schematic illustration of the main parts of our setup used for coaxial electrospinning and of the resulting composite fibres.

geometry. As we will show below, these effects can be quite dramatic, involving substantial expansion of the temperature range of certain phases, while others may disappear.

Liquid crystals (LCs) are fluids with long-range orientational order, rendering them optically anisotropic ${ }^{10}$. In a cholesteric liquid crystal (abbreviated $\mathrm{N}^{*}$, reflecting that it is a chiral version of the nematic LC phase) this order, locally described by the director $\mathbf{n}$, is helically modulated along an 


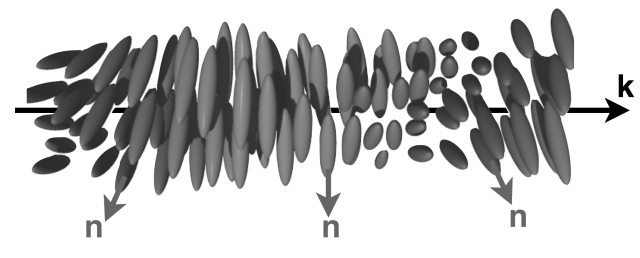

Fig. 2 Cartoon of how the orientational order in a cholesteric liquid crystal consisting of rod-like molecules is helically modulated along an axis $\mathbf{k}$ that is everywhere perpendicular to the director $\mathbf{n}$.

axis $\mathbf{k} \perp \mathbf{n}$ as schematically shown in Fig. 2. Because the director defines the local optic axis also the refractive index for any given polarisation is periodically modulated. In case of short-pitch cholesterics (such as the one used in this work) where the periodicity of the helix is in the range of visible light wavelengths in the medium, this means that the LC acquires the properties of a 1D chiral photonic crystal ${ }^{11}$.

The transmission of light passing along $\mathbf{k}$ is blocked within a narrow wavelength band centred around the value of the optical periodicity if it is circularly polarised with the same handedness as the cholesteric helix. In contrast, the opposite circular polarisation passes unobstructed through the LC. If illuminated with natural unpolarised light, the cholesteric splits up the light within this wavelength band in two oppositely circular-polarised components, transmitting half of the light but strongly reflecting the other half, having the circular polarisation equivalent to the helix handedness. This gives cholesteric LCs beautiful and characteristic iridescent colours.

Because the helix pitch and thus the optical periodicity depends sensitively on several external factors cholesteric LCs are highly useful in sensors and indicators. While temperature monitoring currently is the most common commercial use they have potential for application in e.g. gas sensing ${ }^{12}$. Cholesteric liquid crystals are thus highly useful functional materials that one may wish to incorporate in a variety of products. By electrospinning them coaxially inside a thin polymer sheath we here produce non-woven fibre mats that exhibit the functionality and responsiveness of these chiral LCs. Moreover, it turns out that the liquid crystalline properties of the core fluid are also beneficial for the electrospinning process in itself, stabilising the coaxial morphology in situations where an isotropic fluid core would lead to jet break-up and largescale phase separation. The resulting fibres constitute an entirely new configuration for applying as well as studying liquid crystals, opening possibilities very different from standard LC device configurations, most conspicuously for the development of smart textiles/wearable electronics. While fibres with cholesteric cores have been produced previously by conventional melt spinning and extrusion ${ }^{13}$, this is the first time that electrospinning is used, resulting in a fibre diameter on the order of a micron, that is, two orders of magnitude smaller.
The wall thickness is typically in the range of $100-300 \mathrm{~nm}$.

\section{Results and Discussion}

Since we wish our fibres to exhibit the optical properties of the $\mathrm{N}^{*}$ core it is important that the polymer sheath is transparent, optically isotropic and induces a minimum of scattering. We meet the first two requirements quite well by using poly(vinyl pyrrolidone)_-PVP_as sheath material, essentially solidifying in an amorphous state (the electrospinning process actually results in a weak birefringence of the PVP sheath, albeit much lower than that of the liquid crystal). The final criterion would require refractive index matching to the core, a goal that is more challenging. As a compromise we keep the sheath thin by using a rather dilute polymer solution (12.5 wt.\% PVP in ethanol) for our spinning experiments.

At first sight it might seem surprising that we succeed in obtaining a stable coaxial jet and well-formed core-sheath fibres using this sheath solution, because previous experiments with isotropic low molecular mass core fluids spun inside pure PVP solutions resulted either in structural break-up and deposition of droplets of the core liquid next to solid fibres of pure PVP 5 or in strongly irregular fibres where the core fluid was concentrated inside beads along the fibres rather than in a continuous cylindrical core ${ }^{9}$. These normally unwanted scenarios can be understood as a result of the Rayleigh instability promoting a separation of a jet of a Newtonian fluid into droplets in order to minimise the interfacial tension. Together with strong differences in elongational viscosity between core and sheath fluids this may lead to beading and eventually to complete phase separation. In our case, however, the core fluid is liquid crystalline at room temperature (at which all experiments were carried out) and it is therefore in fact weakly non-Newtonian, allowing the extraction of comparatively long filaments from a drop of the liquid without structural break-up. We propose that this property supports the coaxial jet structure sufficiently to allow the stability of the core-sheath structure throughout the electrospinning process.

Our cholesteric LC reflects selectively in the band 440 to $480 \mathrm{~nm}$, giving it a distinctly blue colour when viewed in reflection (in contrast to many cholesterics the helical pitch is in this mixture close to temperature-independent). On heating, the $\mathrm{N}^{*}$ phase is replaced by a so-called blue phase $\left(\mathrm{BP}^{*}\right)$ at $28.5^{\circ} \mathrm{C}$, succeeded by the isotropic liquid phase at $30.2^{\circ} \mathrm{C}$ (the clearing point). Blue phases are subtle liquid crystal phases (there are three in total) that sometimes appear between the cholesteric and the isotropic phase ${ }^{14,15}$. They are characterised by very strong helical twisting along any radial direction around a central director (perpendicular to all twist axes). The resulting geometry is referred to as double twist cylinders, which on a large scale pack into a cubic lattice in two of the phases, the third being amorphous. In contrast to ordi- 


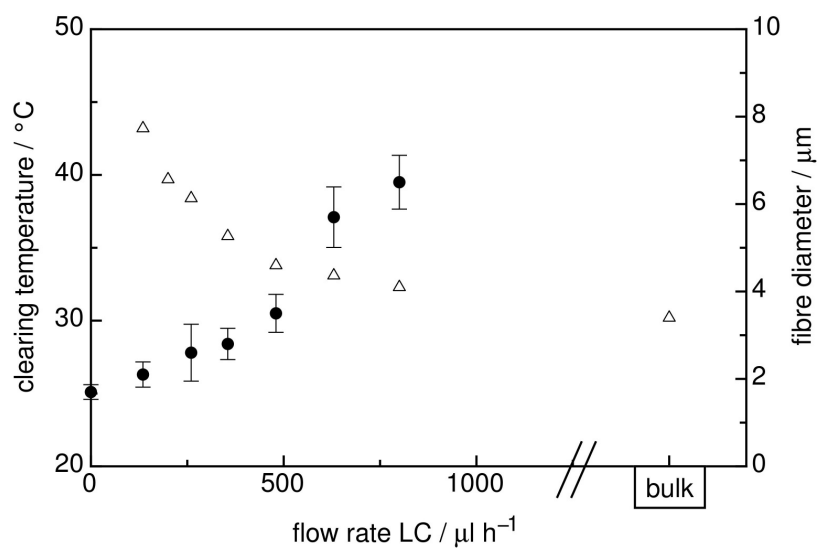

Fig. 3 Outer fibre diameter (filled circles), obtained as an average of 25-30 measurements at different locations within a mat, and clearing point (empty triangles), as obtained by DSC, of the encapsulated as well as the bulk liquid crystal as a function of LC flow rate. The polymer sheath solution flow rate is kept constant at $1.8 \mathrm{ml} \mathrm{h}^{-1}$.

nary LCs, blue phases have no linear birefringence but just like cholesterics they selectively reflect circularly polarised light. At the end of this article we will see that the balance between cholesteric and blue phases is affected in an interesting way by the encapsulation in thin cylindrical fibres.

We vary the flow rate of the liquid crystal from 0.13 to 0.80 $\mathrm{ml} \mathrm{h}^{-1}$ while keeping all other spinning parameters constant, the polymer sheath solution being pumped at a flow rate of 1.8 $\mathrm{ml} \mathrm{h}^{-1}$ as standard setting. This results in fibres with increasing outer diameter as can be seen in Fig. 3. For low LC flow rates the macroscopic appearance of the fibres is dominated by scattering of the polymer sheath, rendering the spun mat white when viewed without magnification. To distinguish the bright colour of the cholesteric core in these fibres, observation through an optical microscope is required. However, for LC flow rates greater than about $0.7 \mathrm{ml} \mathrm{h}^{-1}$, corresponding to a fibre thickness around $5 \mu \mathrm{m}$, the fibre mat macroscopically takes on the optical properties of the encapsulated cholesteric, the selective reflection being easily discernible by the naked eye as shown in Fig.4 a-b. The left image shows the blue colour seen in reflection whereas part b) shows the same fibre mat in transmission, its colour now being the complementary yellow-brown that results from half the blue light having been selectively reflected away from the viewer. The same result can be obtained with slightly thinner fibres by reducing the outer flow rate to $1.1 \mathrm{ml} \mathrm{h}^{-1}$, yielding macroscopically coloured fibre mats at correspondingly lower LC feed rates.

If the mat is heated above the clearing point of the LC in the fibre core, where this turns into an ordinary isotropic liquid and thus looses its peculiar optical properties, the mat accordingly turns white, as shown in Fig. 4c. This electrospun mat thus has a threshold-type temperature sensing capability.
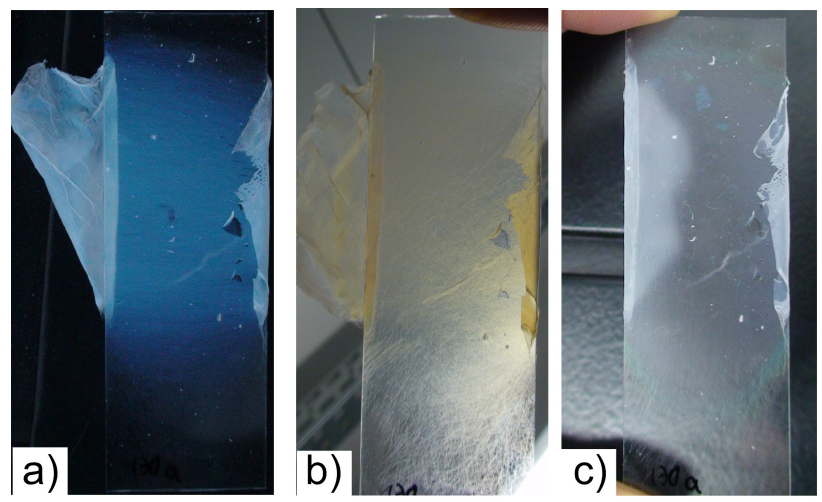

Fig. 4 Fibre mat with the colour of the selective reflection of the LC being visible even macroscopically by the naked eye, viewed in reflection (a), transmission (b) and reflection after heating the $\mathrm{LC}$ to the isotropic phase (c).

A careful look at the photo reveals, however, that some blue areas remain. The reason is that the encapsulation of the $\mathrm{LC}$ in the fibres may affect the temperature range of LC phases considerably, as we also found previously for nematic ${ }^{7}$ and smectic $^{8}$ LCs in coaxially electrospun fibres. As in these cases, the clearing point is higher than in the bulk mixture, the difference being greater the lower the LC flow rate and the corresponding fibre thickness, cf. Fig. 3. From literature it is known that for coaxial electrospinning a decrease of inner flow rate corresponds especially to a decrease of core diameter ${ }^{5}$, the encapsulation of the LC consequently becoming stronger, leading to this effect. While one might argue that contamination from the solvent or polymer used for the sheath solution could also influence the liquid crystal phase sequence, this is highly unlikely as the polar sheath solution is immiscible with the nonpolar liquid crystal. Moreover, any such contamination would decrease, not increase, the clearing point.

For the thinnest fibres the shift in clearing point leads to an extension of the $\mathrm{N}^{*}$ temperature range of about $15 \mathrm{~K}$ compared to the bulk sample. Within a mat there is a certain polydispersity in terms of fibre diameter, hence we may conclude that the remaining blue regimes in Fig. $4 \mathrm{c}$ have a fibre thickness that is slightly lower than the average for the mat, the encapsulated LC there consequently having a somewhat higher clearing point.

Studies on confinement effects on liquid crystal phase transitions have often been carried out with very much stronger confinement, e.g. in porous media with pore size on the order of $10 \mathrm{~nm}^{16}$, hence these are not directly comparable with our situation. The main effect detected in those studies was a smearing-out of the phase transition, making a definition of a clearing point difficult. Here, we see some smearing-out as well but the main effect on the clearing transition is the shift in temperature. Moreover, in contrast to the bulk LC behaviour 


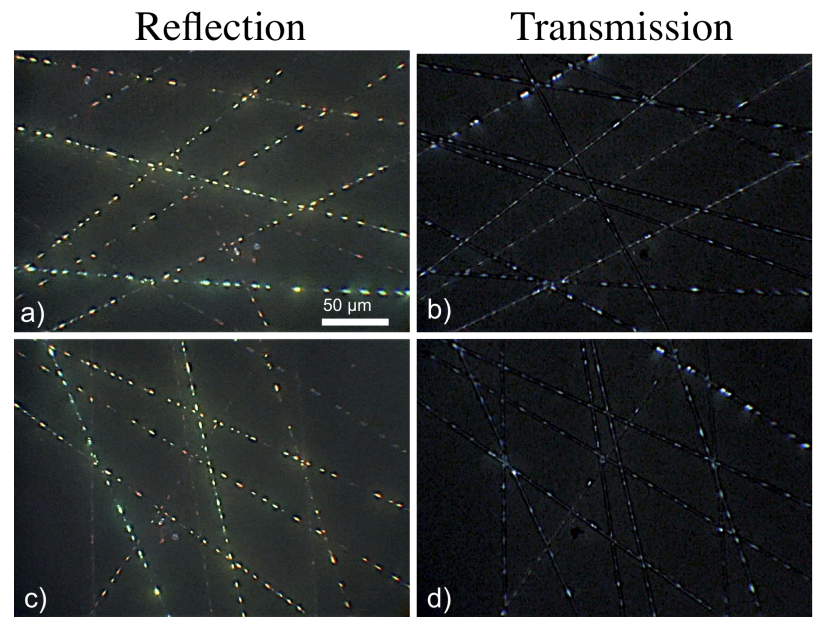

Fig. 5 Polarising microscopy photos of the punctuated texture found in the thinnest fibres showing selective reflection in all colours (a,c) and weak transmission (b, d). For photos $c$ and $d$ the sample has been rotated compared to $a$ and $b$, revealing close to no orientation dependence in reflection as well as in transmission. The flow rates used to produce these fibres were $1.8 \mathrm{ml} \mathrm{h}^{-1}$ for the polymer solution and $0.13 \mathrm{ml} \mathrm{h}^{-1}$ for the liquid crystal.

we never found any inter-LC phase transition in the fibres, no matter what fibre thickness. Neither by polarising microscopy nor by DSC can any phase transition between $\mathrm{N}^{*}$ and $\mathrm{BP} *$ be detected on heating or on cooling. We will come back to an explanation of this phenomenon at the end of the paper.

The appearance of the fibres in polarising microscopy turns out to be more complex than for our previous non-chiral samples $^{7,8}$, a range of textures and optical behaviours being identified when investigating different $\mathrm{N}^{*}$-containing samples. Most common is selective reflection at a colour and intensity that are independent of the orientation of the fibre with respect to the polarisers, allowing it to be easily distinguished from birefringence effects (which must effectively disappear once per $90^{\circ}$ rotation of the sample, every time the optic axis is parallel to one of the polarisers). When viewing the fibre in transmission we see the same colour, again irrespective of sample orientation, due to an effect that may be called 'selective transmission': because light in the reflection band is circularly polarised the transmitted component passes through the analyser of the microscope regardless of sample orientation. Often this selective transmission colour is overlaid with birefringence.

On the whole, three main configurations can be distinguished, of which two stand out as more recurrent:

1. At low LC feed rates, thus thin fibres, the core is not continuous but the LC seems to separate into elongated drops inside the fibres, resulting in a punctuated texture, cf. Fig. 5. The LC in these drops selectively reflects
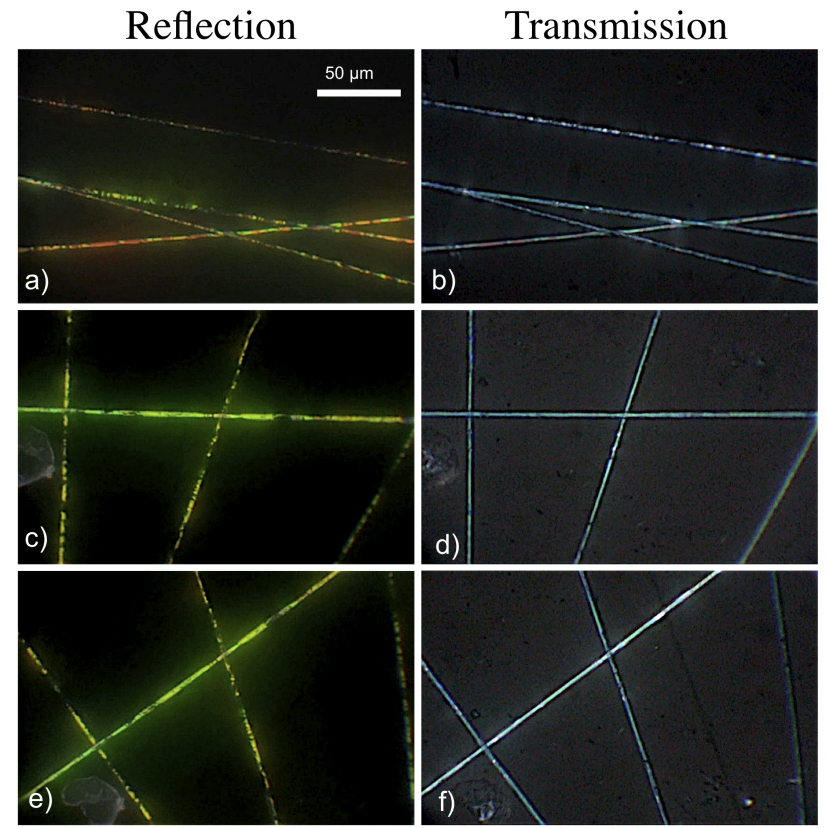

Fig. 6 Polarising microscopy photos of the semicontinous texture found in fibres with medium to large diameter. The first row shows the same sample position in reflection (a) as well as transmission (b), strongly coloured red and yellow sections being most pronounced in the former due to the effect of overlaid birefringence in transmission. Pictures $\mathrm{c}$ - $\mathrm{f}$ show another area of the same sample with greenish selective reflection $(c, e)$, the most common in the thickest fibres. In transmission $(\mathrm{d}, \mathrm{f})$ the colour is again weaker due to overlaid birefringence. These fibres were spun with $1.1 \mathrm{ml} \mathrm{h}^{-1}$ outer and $0.63 \mathrm{ml} \mathrm{h}^{-1}$ inner flow rate.

in various colours ranging from red to blue, sometimes in one and the same fibre. The drops exhibit selective transmission that in some cases is very weak. Birefringence only rarely occurs, then without selective reflection/transmission.

2. At medium to large feed rates, yielding thicker fibres, a semi-continuous to continuous LC core is the result, cf. Fig. 6. This shows selective reflection/transmission at all colours of the spectrum, yellow to blue being most common, overlaid with weak birefringence.

3. A few fibres are quite strongly birefringent without any selective reflection/transmission, with the optic axis along the fibre axis, cf. Fig.7.

Before explaining the observations of different texture types it is helpful to first briefly consider what director configurations we may expect for an $\mathrm{N}^{*}$ phase encapsulated inside a very thin cylindrical fibre. In principle we may distinguish three distinctly different cases as illustrated in Fig. 8. First, (Fig. 8a), the helix could be unwound, a scenario one might 

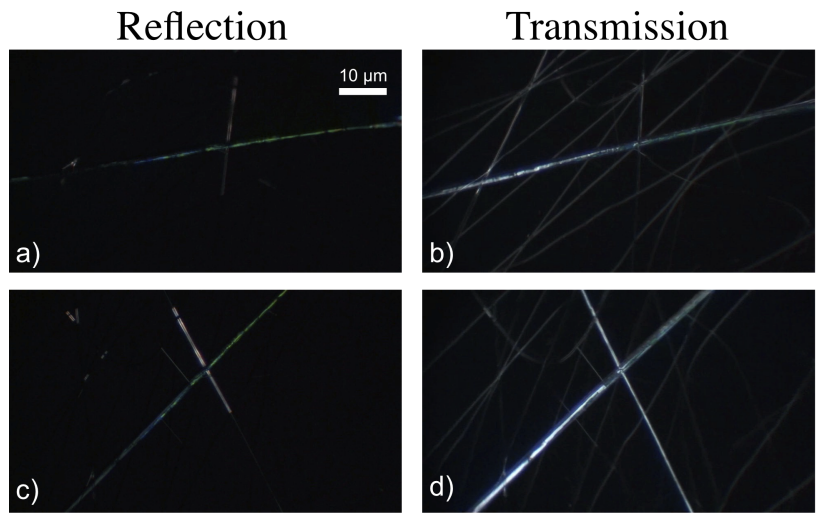

Fig. 7 Example of one of the quite rare fibre samples exhibiting strong birefringence but no selective reflection/transmission. The sample has been rotated between $\mathrm{a}-\mathrm{b}$ and $\mathrm{c}-\mathrm{d}$ to demonstrate the orientation dependence of the texture both in reflection and transmission.

expect for strong surface anchoring and extremely thin fibres and/or long pitch. The latter is certainly not our case but strong surface anchoring with $\mathbf{n}$ along the fibre (planar anchoring) is expected from our previous studies of achiral LCs in fibres of similar composition ${ }^{7,8}$.

On the other hand, the same director alignment at the interface to the polymer sheath is compatible with a helical structure with $\mathbf{k}$ perpendicular to the fibre (Fig. 8c) if the inner fibre diameter is on the order of the natural $\mathrm{N}^{*}$ pitch length or greater (as will be explained below the inner fibre diameter must be an integer number of full helix pitch lengths). This configuration would produce selective reflection/transmission, corresponding to the most commonly occurring texture. Hence, we must in fact have essentially the structure of Fig. 8c in the majority of fibres. With the shortpitch $\mathrm{N}^{*}$ phase used here we would need substantially thinner fibres to obtain the unwound configuration, but if one would let the cholesteric pitch increase strongly the configuration in Fig. 8a would eventually be expected. Our cholesteric mixture has a close to temperature-insensitive pitch but $\mathrm{N}^{*}$ phases with a pitch that diverges on cooling are common, hence fibres containing such phases may change between the configurations in Fig. $8 \mathrm{c}$ and a) as a function of temperature.

While the optical appearance of a fibre with director configuration as in Fig. 8c should be dominated by selective reflection/transmission, the fibre will also be birefringent since $\mathbf{k}$ rotates radially around the fibre, being entirely in the sample plane at the longitudinal cross section at mid fibre height (sketch at the top of Fig. 8c). This explains why also the selectively reflecting fibres typically exhibit weak birefringence as well, in contrast to standard planar-aligned $\mathrm{N}^{*}$ samples in flat cells that are viewed more or less along $\mathbf{k}$.

A third possibility is that the helix develops along the fi-

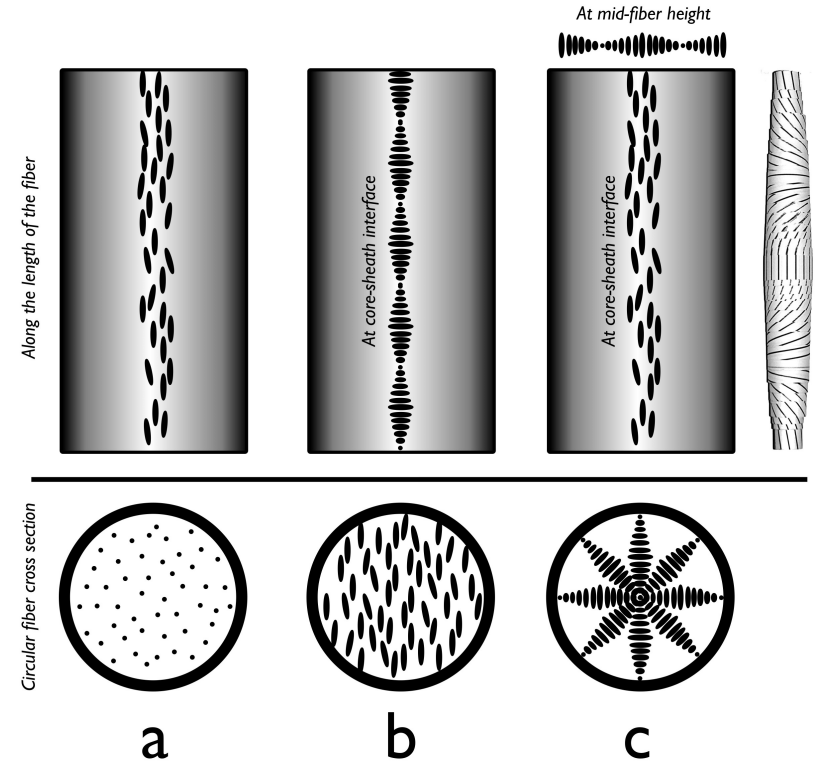

Fig. 8 Schematic illustration of three possible director configurations for an $\mathrm{N}^{*}$ phase encapsulated inside a cylindrical fibre. (a) Planar director anchoring at the core-sheath interface and unwound helix, (b) no or weak director anchoring and helix along the fibre, (c) planar anchoring at the core-sheath interface and helix perpendicular to the fibre. The latter corresponds to the double twist cylinder geometry (right model, lines indicating the director field) that constitutes the basic building block of blue phases.

bre as in Fig. 8b. Since we are then looking perpendicular to a short-pitch helical modulation of the director, the optical result will be strong birefringence and no selective reflection/transmission, hence this configuration would fit with textures like in Fig. 7. Compared to Fig. 8c this structure would be favourable in the sense that the helix has its natural pitch regardless of fibre diameter. It would however require degenerate director alignment at the polymer-LC interface (the structure violates cylindrical symmetry locally, cf. the perpendicular cross section in Fig. 8b), rendering it difficult to reconcile with our previous observations of strong planar director anchoring at the LC-sheath interface. While this configuration cannot be entirely ruled out, possibly being the origin of the relatively rare birefringent but not selectively reflecting/transmitting fibres, we will now see that also the structure in Fig. 8c can explain the behaviour in these fibres.

As can be seen in the cross section drawing in Fig. $8 \mathrm{c}$ the planar anchoring boundary condition requires the inner diameter to be a multiple of the full helix pitch. Note that in contrast to standard flat samples, where the same boundary condition imposes a quantisation into half-pitch units ${ }^{10}$, this is not allowed in the fibre because the cylindrical symmetry requires the director at the centre of the fibre also to be directed along the fibre. Because we have a certain variation of fibre 


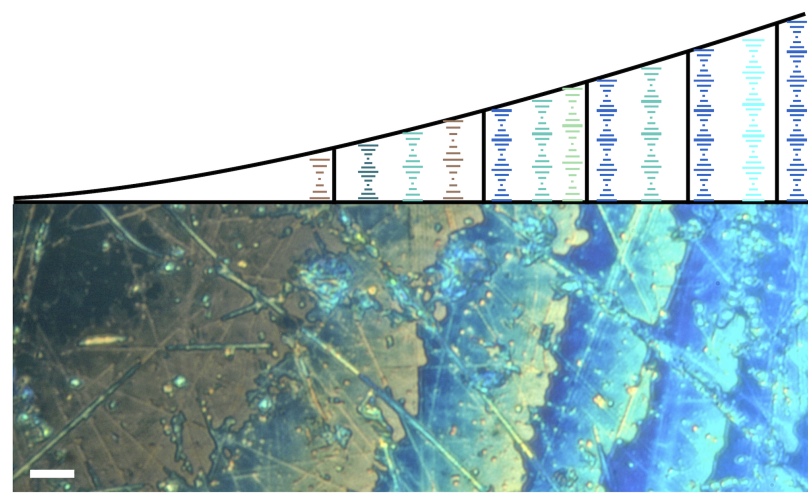

Fig. 9 A so-called 'Cano preparation' of the cholesteric LC used in this work, here enclosed between a flat substrate and a lens (severely scratched, giving rise to many defects), both imposing alignment of $\mathbf{n}$ in the sample plane and along a certain specified direction. To fulfil these boundary conditions the helix is quantised into half-pitch units regardless of distance between the substrates (upper cartoon part). The helix must then be compressed or expanded at any location where the distance is not equal to an integer number of natural half pitches, changing the selectively reflected colour which reaches the longest wavelengths in the thin regime of the sample. Between regions of compression and expansion a defect appears where the number of half-pitch units in the helix changes by one. Scale bar is $10 \mu \mathrm{m}$.

diameter we must then also have a variation in selective reflection/transmission colour, as the pitch must adjust to the actual fibre diameter. The same phenomenon is easily seen in a classical Cano wedge cell geometry ${ }^{10}$, albeit with the quantisation then being in half-pitch steps, as shown in Fig. 9 for the cholesteric LC used in our work. By measuring the ring radii in this photo one can calculate the pitch to be approximately $340 \mathrm{~nm}$, as described in the $\mathrm{ESI}^{\dagger}$.

We indeed see a substantial variation of colour in our fibres, although the LC in bulk is distinctly blue. We can now understand this as a result of the helix quantisation resulting from the cylindrical encapsulation. One notes in Fig. 9, however, that dramatic colour changes result only in case of very small substrate separation, allowing only a single helix 'quantum' to develop between the substrates. Since we in fact see red colour from our originally blue $\mathrm{N}^{*}$ phase once encapsulated in the fibres we understand that the core diameter of our fibres must be on the order of a single helix pitch. These ideas can easily be quantified by plotting the reflection colour, calculated according to the criteria just defined (see ESI for further details), as a function of fibre diameter for a certain natural helix pitch, cf. Fig. 10.

For our case of an $\mathrm{N}^{*}$ phase with a natural helix pitch $p_{0} \approx 340 \mathrm{~nm}$, reflecting in the blue (the average refractive index of about 1.36, established in the ESI, reduces the wavelength of blue light to this value inside the $\mathrm{N}^{*}$ phase), the

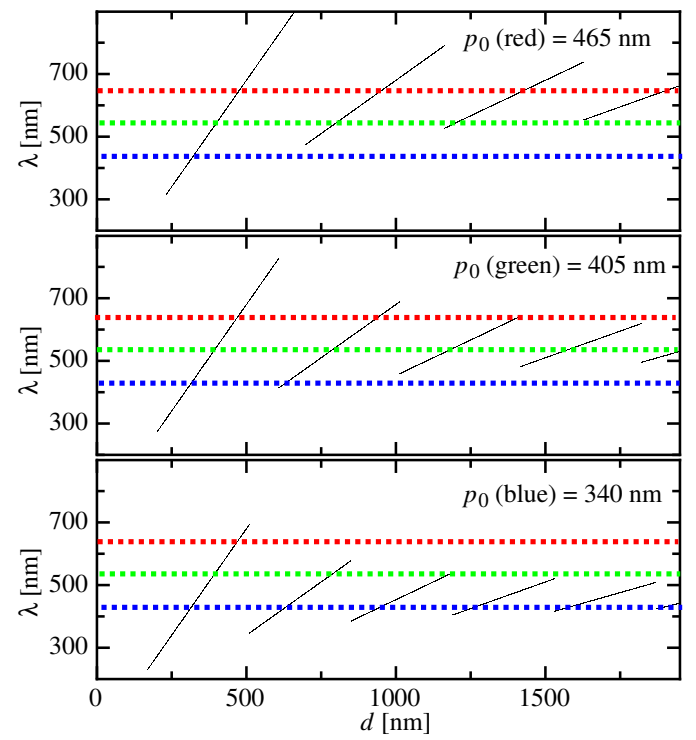

Fig. 10 Selective reflection wavelength $\lambda$ from a cholesteric LC confined in a cylindrical fibre as in Fig. 8c, calculated for three different values of the natural pitch $p_{0}$ that the $\mathrm{N}^{*}$ phase adopts in a bulk sample and plotted as a function of the inner diameter $d$. As a guide to the eye blue, green and red dotted lines have been drawn roughly at the wavelengths corresponding to these colours.

lower diagram in Fig. 10 corresponds to our fibres. We immediately see that a red colour can arise only for an inner diameter of about $480 \mathrm{~nm}$, neither less nor more, hence we know that this is the inner diameter of the fibre sections displaying this colour, e.g. in Fig. 6a. On the other hand, the majority of fibres with continuous LC filling show yellow-green colour, i.e. reflection at wavelengths around $500-550 \mathrm{~nm}$. There are three core diameter ranges that would produce this colour, $\sim 400 \mathrm{~nm}, \sim 800 \mathrm{~nm}$ and (just barely) $\sim 1180 \mathrm{~nm}$. The first and last of these ranges are highly unlikely since we should have seen much more red-coloured fibres should the thinnest range apply, and since blue would have been much more frequently encountered if the larger range would occur commonly. We can thus conclude that the best filled fibres have a core diameter in the range around $\sim 800 \mathrm{~nm}$.

An important piece of information in Fig. 10 is that the wavelength that is reflected from our $\mathrm{N}^{*}$ phase in the electrospun fibres is in the UV range for many fibre diameters (e.g. for core diameters in the range $515-640 \mathrm{~nm}$, a range that we from the above considerations know must be represented in our samples). These fibres will thus not produce any visibly reflected colour, hence the only optical effect that should be seen in these fibres is birefringence due to components of $\mathbf{k}$ in the sample plane. In other words, this may be an explanation of the purely birefringent fibres (Fig. 7) that is more plausible 


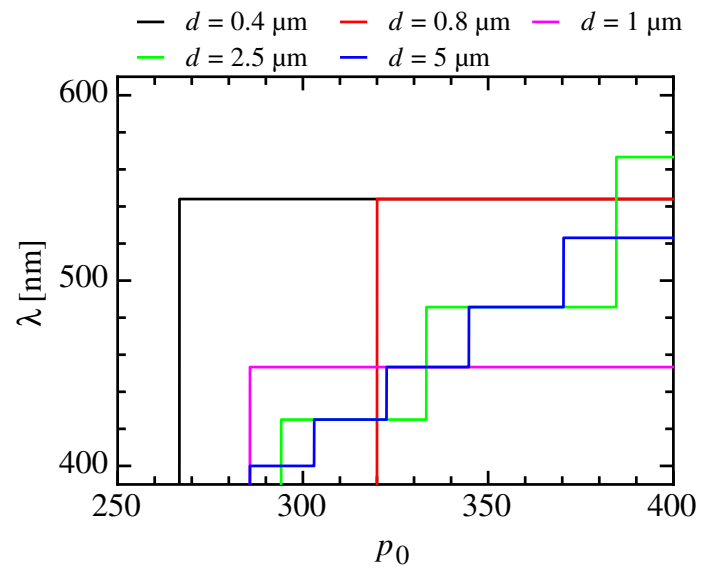

Fig. 11 Selective reflection wavelength $\lambda$ from a cholesteric LC confined in a cylindrical fibre as in Fig. 8c, calculated for five different values of inner diameter $d$ and plotted as a function of the natural pitch $p_{0}$ that the $\mathrm{N}^{*}$ phase adopts in a bulk sample.

than the configuration in Fig. 8b.

We may also plot the same relation between reflection wavelength $\lambda$, natural helix pitch $p_{0}$ and inner diameter $d$ of the confining cylinder as a function of $p_{0}$ for certain values of $d$, cf. Fig. 11. We then see that for core diameters around $1 \mu \mathrm{m}$ the colour is in fact almost entirely determined by the confinement, any tendency of the cholesteric to change its pitch having essentially no effect. This means that fibres that should be able to display colour change, e.g. as a function of temperature in textiles with thermometer functionality, should have an inner diameter no less than some $5 \mu \mathrm{m}$. On the other hand, for ON-OFF sensing applications such as gas detection it may be more desirable to fix the basic colour by relatively strong confinement. By adding a reactive species to the $\mathrm{LC}$ that changes or even expels the helix when it comes into contact with the analyte at a concentration above a threshold ${ }^{12}$, this would trigger a discrete colour change, or even complete disappearance of colour, that is easy to recognise. Obviously, easy detection then requires optimisation of the sheath to reduce scattering to the extent that the colour from these thin fibres is easily visible by the naked eye.

The most bewildering texture category is the one of the elongated droplets (Fig. 5), especially its commonly occurring contrast between strong reflection and weak transmission. Without any aim to give a solid explanation, we may speculate that the apparent general darkness of the droplets when viewed in transmission is related to the more complex optical situation of a liquid crystal in droplet geometry, resulting in greater variation of $\mathbf{k}$ as well as stronger refraction effects arising from the curvature in all directions as well as the varying sheath thickness. Moreover, the general reduction in core thickness in these fibres obviously renders the effective bire- fringence smaller.

One may also ask what the origin of the separation into droplets is. While it on the one hand could have happened during the electrospinning process, one might then have expected a beaded outer fibre morphology, something we did not see. On the other hand, a thin but continuous inner core may initially have been formed in the spinning process, this then transforming into droplet morphology soon after fibre formation in order to reduce the interfacial area between the nonpolar LC and the polar sheath polymer. This would require that the sheath still contained a sufficient amount of solvent to be soft enough to accommodate the morphology change, an assumption that is not unreasonable directly after fibre deposition. It also correlates with the observation that flow within the liquid crystalline fibre core could often be detected directly after deposition, at least for thicker fibres clearly visible in the optical microscope as spontaneous textural changes.

Finally, we come back to the absence of the $\mathrm{N}^{*}$-BP* transition of the LC within our fibres. Having argued that the most common director configuration in our fibres is that in Fig. 8c we note that this is an unusual structure for the $\mathrm{N}^{*}$ phase, in which the helix axis $\mathbf{k}$ normally extends only in a single welldefined direction. In fact, the configuration with radial helix arrangement that we have here, imposed onto the $\mathrm{N}^{*}$ director field by the confinement in cylindrically symmetric fibres, is nothing else than the double-twist cylinder configuration that is the basic building block for blue phases (see model on the far right of Fig. 8). The same situation was achieved by Kitzerow et al. ${ }^{17}$ by filling $\mathrm{N}^{*} \mathrm{LCs}$ into glass capillaries, albeit on an order of magnitude larger scale. Both the cholesteric pitch and the capillary diameter were typically in the range tens of microns. The $\mathrm{N}^{*}$ director configuration was thus in their case structurally analogous to the $\mathrm{BP}^{*}$ double-twist cylinder but the scale was entirely different. In our case both the structure and the scale of the $\mathrm{N}^{*}$ director configuration inside the fibres is equivalent to the $\mathrm{BP}^{*}$ phase building block, hence the two phases are effectively degenerate in the electrospun fibres. This explains why we no longer see any $\mathrm{N}^{*}$-BP* transition in the fibres. For even thicker fibres we would eventually expect to see the transition again, as multiple double-twist cylinders could build up a cubic network in the $\mathrm{BP}^{*}$ phase once the cylindrical confinement is less strong.

\section{Conclusions and Outlook}

In this work we have demonstrated that microfibres that selectively reflect light of a certain colour in the visible spectrum can easily be produced by coaxial electrospinning of a cholesteric liquid crystal inside a polymer sheath. When the LC flow rate is high, approaching the flow rate of the outer polymer solution, the fibre mats show macroscopically the colour that is selectively reflected by the cholesteric core. In 
case of lower LC flow rate the core diameter is thinner, resulting in quantisation effects that may change the reflected colour substantially away from the natural colour of the bulk LC. An interesting future venture is to verify the proposed director configuration in the fibers by means of microscopic techniques with 3D capabilities of director reconstruction, such as fluorescent confocal polarizing microscopy (FCPM) ${ }^{18}$ or coherent anti-Stokes Raman scattering (CARS) ${ }^{19}$.

By using selected LC materials, showing different colours of selective reflection and different degrees of temperature sensitivity of this colour, a new class of functional composite fibres can be simply produced with our method, with potential for intelligent non-woven textiles with e. g. integrated sensing functionality. Depending on the intended application, thin fibres with sub micron core diameters may be spun, resulting in colour quantisation effects useful for sensors with ON-OFF response type, whereas fibres with some $5 \mu \mathrm{m}$ core diameter can change their colour all the way from red to blue as a response to changes in the environment, convenient e.g. for temperature sensing. The encapsulation of the LC also leads to broadening of the LC phase and the cylindrical geometry imposes an unusual director configuration to the cholesteric phase making it degenerate with blue phases.

\section{Experimental}

The LC used in this work was a 50:50 mixture (by weight) of the chiral dopant (S)-4-cyano-4-(2-methylbutyl)-biphenyl (Synthon Chemicals) and the nematic mixture RO-TN403/015S (Hoffmann-La Roche), with a resulting phase sequence of $\mathrm{N}^{*} 28.5 \mathrm{BP}^{*} 30.2$ Iso. The fibres were spun with the LC in its cholesteric phase at room temperature. As outer polymer solution $12.5 \mathrm{wt} . \%$ PVP (MW $1.300 .000 \mathrm{~g} / \mathrm{mol}$, Acros) and $0.5 \mathrm{wt} . \% \mathrm{NaCl}$ were dissolved in ethanol.

For the fibres discussed here, the LC flow rate was varied from 0.13 to $0.8 \mathrm{ml} \mathrm{h}^{-1}$ and the outer flow rate was 1.1 or, most commonly, $1.8 \mathrm{ml} \mathrm{h}^{-1}$. The two solutions were pumped by a microfluidics control unit (Fluigent MFCS-4C) through a metallic tube and a flexible capillary (fused silica tubing, BGB Analytik AG), respectively. The latter (inner diameter $0.25 \mathrm{~mm}$, outer diameter $0.35 \mathrm{~mm}$ ) was inserted coaxially into the former (inner diameter $1.0 \mathrm{~mm}$ ), as shown in Fig. 1. An electric voltage of $10 \mathrm{kV}$ was applied with a high voltage power supply (Gamma High Voltage Research) to the spinneret, the grounded collector electrode being set $10 \mathrm{~cm}$ below the spinneret.

Phases and phase transitions of the LC were determined by polarising optical microscopy (Nikon microscope equipped with a Mettler hot stage) and by differential scanning calorimetry (Perkin Elmer Pyris 1 at a heating / cooling rate of $10 \mathrm{~K} \mathrm{~min}^{-1}$ ), respectively.

\section{Acknowledgments}

Financial support from the "Excellenzcluster nanostrukturierte Materialien" of the Land Sachsen-Anhalt is gratefully acknowledged. D. Krüerke is thanked for his helpful suggestions in realizing the Cano experiment.

\section{References}

1 A. Greiner and J. Wendorff, Adv. Polym. Sci., 2008, 219, 107-171.

2 D. Reneker and A. Yarin, Polymer, 2008, 49, 2387-2425.

3 C. Burger, B. Hsiao and B. Chu, Ann. Rev. Mater. Res., 2006, 36, 333368.

4 D. Li, Y. Wang and Y. Xia, Adv. Mater., 2004, 16, 361-366.

5 D. Li, A. Babel, S. Jenekhe and Y. Xia, Adv. Mater, 2004, 16, 2062.

6 I. Loscertales, A. Barrero, M. Marquez, R. Spretz, R. Velarde-Ortiz and G. Larsen, J. Am. Chem. Soc., 2004, 126, 5376-5377.

7 J. P. F. Lagerwall, J. T. McCann, E. Formo, G. Scalia and Y. Xia, Chem. Commun., 2008, 5420-5422.

8 E. Enz, U. Baumeister and J. Lagerwall, Beilstein J. Org. Chem., 2009, 5, DOI: $10.3762 /$ bjoc.5.58.

9 J. Díaz, A. Barrero, M. Márquez and I. Loscertales, Adv. Funct. Mater., 2006, 16, 2110-2116.

10 P.-G. de Gennes and J. Prost, The physics of liquid crystals, Clarendon Press, Oxford, UK, 1993.

11 H. De Vries, Acta Cryst., 1951, 4, 219-226.

12 Y. Han, K. Pacheco, C. W. M. Bastiaansen, D. J. Broer and R. P. Sijbesma, J. Am. Chem. Soc., 2010, 132, 2961-2967.

13 M. Nakata, M. Sato, Y. Matsuo, S. Maeda and S. Hayashi, J. SID, 2006, 14, 723-727.

14 P. P. Crooker, Liq. Cryst., 1989, 5, 751-775.

15 H. S. Kitzerow, ChemPhysChem, 2006, 7, 63-66.

16 G. Iannacchione, G. Crawford, S. Qian, J. Doane and D. Finotello, Phys. Rev. E, 1996, 53, 2402-2411.

17 H. Kitzerow, B. Liu, F. Xu and P. Crooker, Phys. Rev. E, 1996, 54, 568575.

18 I. Smalyukh, R. Pratibha, N. Madhusudana and O. Lavrentovich, Eur. Phys. J. E, 2005, 16, 179-191.

19 A. Kachynski, A. Kuzmin, P. Prasad and I. Smalyukh, Appl. Phys. Lett., 2007, 91, 151905. 